\title{
STRUCTURE OF DARK MATTER HALO
}

\author{
TOSHIYUKI FUKUSHIGE AND JUNICHIRO MAKINO \\ College of Arts and Sciences, Univ. of Takyo \\ 3-8-1 Komaba, Meguro-ku, Tokyo 153, Japan \\ fukushig@chianti.c.u-tokyo.ac.jp
}

We performed N-body simulation on special-purpose computer, GRAPE4 , to investigate the structure of dark matter halos (Fukushige, T. and Makino, J. 1997, ApJL, 477, L9). Universal profile proposed by Navarro, Frenk, and White (1996, ApJ, 462, 563), which has cusp with density profiles $\rho \propto r^{-1}$ in density profile, cannot be reproduced in the standard Cold Dark Matter (CDM) picture of hierarchical clustering. Previous claims to the contrary were based on simulations with relatively few particles, and substantial softening. We performed simulations with particle numbers an order of magnitude higher, and essentially no softening, and found that typical central density profiles are clearly steeper than $\rho \propto r^{-1}$, as shown in Figure 1. In addition, we confirm the presence of a temperature inversion in the inner $5 \mathrm{kpc}$ of massive galactic halos, and give a natural explanation for formation of the temperature structure.

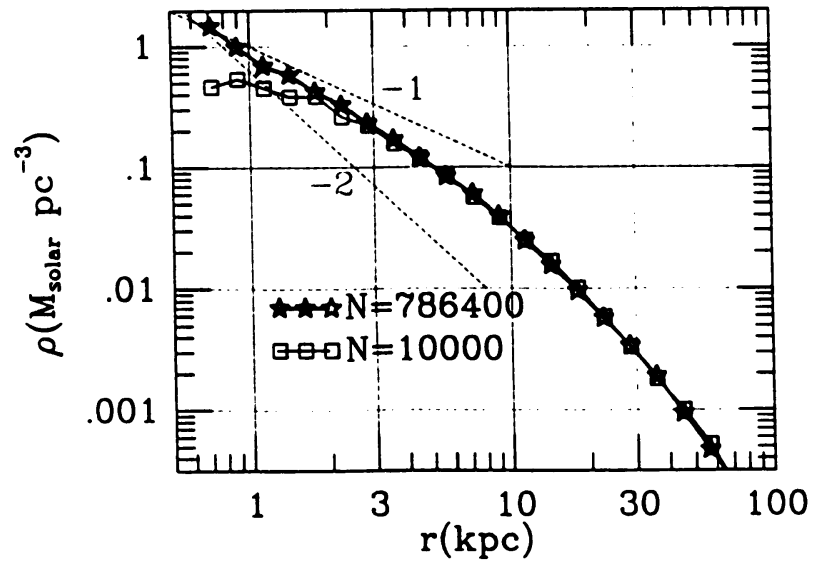

Figure 1. Density profile of formed dark matter halo 